\title{
JACKSON SUMMATION OF THE FABER DEVELOPMENT*
}

\author{
W. E. SEWELL
}

1. Introduction. The purpose of this note is to prove the following theorem:

THEOREM. Let $C$ be an analytic Jordan curve in the z-plane, and let $f(z)$ be analytic in $C$, continuous in $\bar{C}$, the closed limited set bounded by $C$, and let $\dagger f^{(p)}(z),(p \geqq 0)$, satisfy a Lipschitz condition $\ddagger$ of order $\alpha$, $(0<\alpha \leqq 1)$, on $C$. Then

$$
\left|f(z)-\sum_{\nu=0}^{n} d_{n \nu} a_{\nu} P_{\nu}(z)\right| \leqq \frac{M}{n^{p+\alpha}}, \quad \quad z \text { in } \bar{C},
$$

where $M$ is a constant independent of $n$ and $z$,

$$
\sum_{\nu=0}^{n} a_{\nu} P_{\nu}(z)
$$

is the sum of the first $n+1$ terms of the development of $f(z)$ in the Faber $\$$ polynomials belonging to $C$, and $d_{n \nu}$ is the Jackson\| summation coefficient of order $p$.

In a previous paper the author $\uparrow$ showed that under the above hypothesis

$$
\left|f(z)-\sum_{0}^{n} a_{\nu} P_{\nu}(z)\right| \leqq M\left(\log n / n^{p+\alpha}\right), \quad z \text { in } \bar{C} .
$$

Later John Curtiss** proved the existence of a sequence of polynomials $Q_{n}(z)$ of respective degrees $n,(n=1,2, \cdots)$, such that

$$
\left|f(z)-Q_{n}(z)\right| \leqq M / n^{p+\alpha} .
$$

* Presented to the Society, December 30, 1937.

$\dagger f^{(p)}(z)$ denotes the $p$ th derivative of $f(z) ; f^{(0)}(z) \equiv f(z)$.

$\ddagger f(z)$ satisfies a Lipschitz condition of order $\alpha$ on $C$ if for $z_{1}$ and $z_{2}$ arbitrary points on $C$ we have $\left|f\left(z_{1}\right)-f\left(z_{2}\right)\right| \leqq L\left|z_{1}-z_{2}\right|^{\alpha}$, where $L$ is a constant independent of $z_{1}$ and $z_{2}$.

§ G. Faber, Mathematische Annalen, vol. 57 (1903), pp. 389-408.

II Dunham Jackson, Transactions of this Society, vol. 15 (1914), pp. 439-466; p. 463.

I This Bulletin, vol. 41 (1935), pp. 111-117; this paper will be referred to hereafter as SI.

** This Bulletin, vol. 42 (1936), pp. 873-878. 
In a paper soon to appear, Walsh and the author state that for $p=0$, $(0<\alpha<1)$, the inequality $\left|f(z)-\sigma_{n}(z)\right| \leqq M / n^{\alpha}$ is valid, where $\sigma_{n}(z)$ is the $n$th arithmetic mean of the development of $f(z)$ in the Faber polynomials belonging to $C$. Here we extend this result by exhibiting a set of polynomials (proved by Curtiss to exist) with the prescribed degree of convergence for arbitrary $p$ and for $0<\alpha \leqq 1$.

2. Proof of the theorem. Let

$$
z=\psi(t)=\frac{1}{t}+b_{0}+b_{1} t+b_{2} t^{2}+\cdots=\frac{1}{t}+\mathfrak{B}(t)
$$

map the exterior of $C$ on the region $|t|<r$ of the complex $t$-plane so that the point $z=\infty$ corresponds to the point $t=0$. It follows from the analyticity of $C$ that the right-hand side of (2) converges for $|t| \leqq r^{\prime}, r^{\prime}>r$. The Faber polynomials belonging to $C$ are defined as follows: $P_{n}(z)$ is the polynomial of degree $n$ in $z$ such that the coefficient of $z^{n}$ is unity, and, as a function of $t$ through (2), such that the coefficients are zero for the terms in $t^{-n+1}, t^{-n+2}, \cdots, t^{-1}, t^{0}$; hence $P_{n}(z)=1 / t^{n}+t \mathfrak{B}_{n}(t)$, where $\mathfrak{B}_{n}(t)$ converges for $|t| \leqq r^{\prime}$.

Faber (loc. cit.) and the author (SI) have shown that

$$
\begin{aligned}
f(z) & =\sum_{\nu=0}^{\infty} a_{\nu} P_{\nu}(z), & z \text { in } \bar{C}, \\
& =\sum_{\nu=0}^{\infty} a_{\nu}\left(\frac{1}{t^{\nu}}+t \Re_{\nu}(t)\right)=\sum_{\nu=0}^{\infty} \frac{a_{\nu}}{t^{\nu}}+\sum_{1}^{\infty} c_{\nu} t^{\nu}, & r \leqq|t| \leqq r^{\prime} .
\end{aligned}
$$

It should be noted here that (Faber, loc. cit.) $\left|a_{\nu}\right| \leqq M_{1} r^{\nu}$ and $\left|\mathfrak{B}_{\nu}(t)\right| \leqq M_{2} / r^{\prime \nu}$, where $M_{1}$ and $M_{2}$ are constants.

Now we consider

$$
\begin{aligned}
f(z)-\sum_{\nu=0}^{n} d_{n \nu} a_{\nu} P_{\nu}(z)= & f(\psi(t))-\sum_{\nu=0}^{n} d_{n \nu} a_{\nu}\left(\frac{1}{t^{\nu}}+t \Re_{\nu}(t)\right) \\
= & f(\psi(t))-\sum_{1}^{\infty} c_{\nu} t^{\nu}-\sum_{\nu=0}^{n} d_{n \nu} a_{\nu} / t^{\nu} \\
& -\sum_{\nu=1}^{n} d_{n \nu} a_{\nu} t \Re_{\nu}(t)+\sum_{\nu=1}^{\infty} c_{\nu} t^{\nu} \\
= & f(\psi(t))-\sum_{1}^{\infty} c_{\nu} t^{\nu}-\sum_{\nu=0}^{n} d_{n \nu} a_{\nu} / t^{\nu} \\
& +\sum_{\nu=1}^{n}\left(1-d_{n \nu}\right) a_{\nu} t \Re_{\nu}(t)+\sum_{n+1}^{\infty} a_{\nu} t \Re_{\nu}(t), \quad r \leqq|t| \leqq r^{\prime} .
\end{aligned}
$$


Therefore

$$
\begin{aligned}
\left|f(z)-\sum_{\nu=0}^{n} d_{n \nu} a_{\nu} \mathfrak{B}_{\nu}(z)\right| \leqq & f(\psi(t))-\sum_{1}^{\infty} c_{\nu} t^{\nu}-\sum_{\nu=0}^{n} d_{n \nu} a_{\nu} / t^{\nu} \mid \\
& +\left|\sum_{\nu=1}^{n}\left(1-d_{n \nu}\right) a_{\nu} t \mathfrak{B}_{\nu}(t)\right| \\
& +\left|\sum_{n+1}^{\infty} a_{\nu} t \mathfrak{B}_{\nu}(t)\right|, \quad r \leqq|t| \leqq r^{\prime} .
\end{aligned}
$$

The $\operatorname{sum} \sum_{\nu=0}^{n} d_{n \nu} a_{\nu} / t^{\nu}$ is (SI) the Jackson summation of the first $n+1$ terms of the Taylor development of the function $f(\psi(t))-\sum_{1}^{\infty} c_{\nu} t^{\nu}$ and consequently (Jackson, loc. cit.; Curtiss, loc. cit.)

$$
\left|f(\psi(t))-\sum_{1}^{\infty} c_{\nu} t^{\nu}-\sum_{\nu=0}^{n} d_{n \nu} a_{\nu} / t^{\nu}\right| \leqq \frac{M_{3}}{n^{p+\alpha}}, \quad r \leqq|t| \leqq r^{\prime} .
$$

Also

$$
\begin{array}{rlrl}
\left|\sum_{n+1}^{\infty} a_{\nu} t \mathfrak{B}_{\nu}(t)\right| & \leqq \sum_{n+1}^{\infty}\left|a_{\nu}\right||t|\left|\mathfrak{B}_{\nu}(t)\right| \leqq M_{1} M_{2} r \sum_{n+1}^{\infty}\left(\frac{r}{r^{\prime}}\right)^{\nu} \\
& \leqq \frac{M_{4}}{n^{p+\alpha}}, & |t|=r,
\end{array}
$$

since $r / r^{\prime}<1$. Furthermore*

$$
\left|1-d_{n \nu}\right| \leqq \frac{M_{5} \nu^{p+1}}{n^{p+1}}
$$

consequently

$$
\begin{aligned}
\left|\sum_{\nu=1}^{n}\left(1-d_{n \nu}\right) a_{\nu} t \Re_{\nu}(t)\right| & \leqq M_{1} M_{2} M_{5} \sum_{1}^{n} \frac{\nu^{p+1}}{n^{p+1}}\left(\frac{r}{r^{\prime}}\right)^{\nu} \\
& =\frac{M_{1} M_{2} M_{5}}{n^{p+1}} \sum_{1}^{n} \nu^{p+1}\left(\frac{r}{r^{\prime}}\right)^{\nu} .
\end{aligned}
$$

The series on the right converges, and the proof of the theorem is thus complete.

Georgia School of Technology

* Dunham Jackson, Transactions of this Society, vol. 15 (1914), pp. 439-466. It should be noted here that Jackson's summation coefficients vary with the derivative but not with the order of the Lipschitz condition. 